\title{
Bypassing Multidrug Resistance in Cancer Cells with Biodegradable Polymer Capsules
}

\author{
Yan Yan, Christopher J. Ochs, Georgina K. Such, Joan K. Heath, Edouard C. Nice, and Frank \\ Caruso*
}

The development of multidrug resistance (MDR) to chemotherapeutic agents is a major challenge in effective cancer treatment and contributes to treatment failure in over $90 \%$ of patients with metastatic cancer. ${ }^{[1]}$ The most common underlying mechanism of MDR has been correlated with the overexpression of the ATP binding cassette (ABC) transporters, particularly Pglycoprotein (Pgp) ${ }^{[2]}$ leading to the efflux of many anticancer drugs with consequent drug insensitivity. ${ }^{[3]}$ Much effort has been directed towards developing drugs that either evade efflux or suppress the function of Pgp to reverse MDR and hence restore the susceptibility of resistant cancer cells to multiple anticancer drugs. However, due to the exceptionally broad poly-specificity of Pgp, a significant portion of the compounds in the drug development pipeline have been reported to be Pgp substrates. ${ }^{[3,4]}$ Whilst attempts to reverse MDR through the inhibition of Pgp have been ongoing for the last two decades, and still remain a priority, clinical trials using these inhibitors have shown limited success to date, largely due to the lack of specificity and efficacy. ${ }^{[3,5]}$ Clearly, the benefit of developing useful drug analogues or Pgp inhibitors has yet to be realized, leading to an urgent need for new strategies to improve treatment of MDR cancers.

Nanotechnology offers an unprecedented opportunity for improved drug delivery to cancers. The use of nanocarriers can provide new therapies not achievable with current drug modalities, as the nanoengineered delivery systems can be specifically targeted to cancer cells, can carry a large payload and can accommodate multiple drugs to minimize side effects while enhancing drug efficacy. ${ }^{[6]}$ Very recently, studies have shown that nanocrystals, ${ }^{[7]}$ carbon nanotubes, ${ }^{[8]}$ polymeric nano-conjugates, ${ }^{[9]}$ and inorganic nanoparticles ${ }^{[10]}$ have the potential to treat $A B C$ transporter-

[*] Prof. F. Caruso, Dr. Y. Yan, C. J. Ochs, Dr. G. K. Such Centre for Nanoscience and Nanotechnology

Department of Chemical and Biomolecular Engineering

The University of Melbourne,

Parkville, Victoria 3010 (Australia)

Fax: (+61) 3-8344-4153

E-mail: fcaruso@unimelb.edu.au

A/Prof. J. K. Heath, Prof. E. C. Nice

Ludwig Institute for Cancer Research

Royal Melbourne Hospital

Victoria 3050 (Australia)

Prof. E. C. Nice

Department of Biochemistry and Molecular Biology

Monash University

Clayton, Victoria 3800 (Australia)

[**] This work was supported by the Australian Research Council under the Federation Fellowship and Discovery Project schemes (F.C.) and by the National Health and Medical Research Council (NHMRC) Program Grant 487922 (J.K.H., F.C.). mediated MDR cancer, as the drug-loaded nanocarriers can bypass the efflux pumps, such as Pgp, when they enter cells via endocytosis. Recently, we reported the potential of layer-by-layer (LbL) assembled-polymer capsules as an effective chemotherapeutic delivery system to colorectal cancer cells. ${ }^{[11]}$ However, the application of these capsules to drug resistant cancer cells has not yet been reported.

Herein we report the first investigation of the use of LbLassembled biodegradable microcapsules to circumvent Pgpmediated MDR in colorectal cancer cells (Scheme 1). Using a human primary colorectal carcinoma derived cell line (LIM1899), ${ }^{[12]}$ an MDR cell line, which is highly resistant to both doxorubicin (DOX) and paclitaxel (PTX), has been generated and characterized. Based on the intrinsic fluorescence of DOX, we demonstrate increased intracellular accumulation of drug in MDR cancer cells in response to microcapsules, whereas free drug is significantly excluded. The use of the microcapsules loaded with either DOX or PTX efficiently restores drug sensitivity to the MDR cells, suggesting the general applicability of this system to bypass Pgpmediated MDR. Such capsules, which merge modular LbLassembly with click chemistry, can be readily extended to a wide range of materials, such as other polymer-drug conjugates and biomacromolecules, ${ }^{[13]}$ can exert fine control over drug dose and position, ${ }^{[11 b]}$ can be tailored for intracellular release of drugs ${ }^{[14]}$ and can be further biofunctionalized with targeting moieties. ${ }^{[15]}$ This makes them promising candidates for multifunctional delivery systems that enhance cancer-targeted delivery and release multiple cargos in response to physiological stimuli, bypassing MDR in tumours.

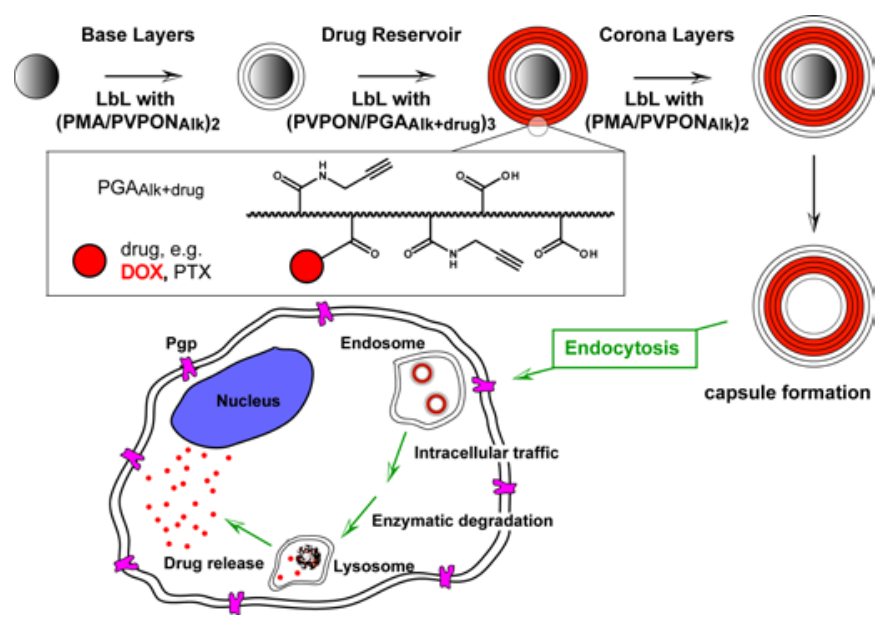

Scheme 1. Schematic diagram showing modular assembly of drugloaded poly(L-glutamic acid) (PGA) capsules and the mechanism by which MDR is bypassed. The structure of the polymer-drug conjugate is shown (expanded). The drug-loaded PGA capsules are internalized by MDR cancer cells via endocytosis. Subsequently incorporated drugs are released by enzymatic degradation in lysosomes. 
The biodegradable microcapsules used here were assembled through a combination of LbL assembly driven by hydrogen bonding and robust click chemistry. ${ }^{[11 b, 16]}$ Firstly, two bilayers of alkyne-functionalized poly(N-vinylpyrrolidone) $\left(\mathrm{PVPON}_{\mathrm{Alk}}\right)$ and poly(methacrylic acid) (PMA) were assembled on colloidal templates. The resulting outer layers of $\mathrm{PVPON}_{\mathrm{Alk}}$ were then hydrogen bonded with alkyne-functionalized poly(L-glutamic acid)drug conjugates ( PGA $_{\text {Alk+drug }}$ ) (The preparation and characterization of polymer-drug conjugates described in Supporting Information). By exploiting the hydrogen bonding between PVPON and $\mathrm{PGA}_{\text {Alk+drug, three layers of PGA }}$ Alk+drug were assembled to create a drug-conjugate reservoir. The LbL assembly was continued to deposit an additional two layers of $\mathrm{PVPON}_{\text {Alk }}$ through hydrogen bonding with PMA. All of the alkyne-functionalized layers were then covalently cross-linked by the copper(I)-catalyzed 1,3cycloaddition of alkynes and azides using a bisazide linker (bisazido dodecaethylene glycol), ${ }^{[11 b]}$ resulting in the formation of $\left(\mathrm{PVPON}_{\mathrm{Alk}}\right)_{2} /\left(\mathrm{PGA}_{\mathrm{Alk}+\mathrm{drug}}\right)_{3} /\left(\mathrm{PVPON}_{\mathrm{Alk}}\right)_{2}$ core-shell particles. After removal of the silica core, the capsules were analyzed with optical microscopy, atomic force microscopy (AFM) and transmission electron microscopy (TEM) (Figure 1). The incorporation of both inner and outer layers of PVPON $_{\text {Alk }}$ rendered the capsules with superior colloidal stability to single-component PGA-drug capsules (data not shown). To demonstrate the versatility of the system, we employed either $\mathrm{PGA}_{\mathrm{Alk}+\mathrm{DOX}}$ or $\mathrm{PGA}_{\mathrm{Alk}+\mathrm{PTX}}$ to generate DOXloaded or PTX-loaded microcapsules, respectively (referred to as PGA-DOX capsules or PGA-PTX capsules). The capsule assembly also allows co-delivery of multiple drugs using this strategy.
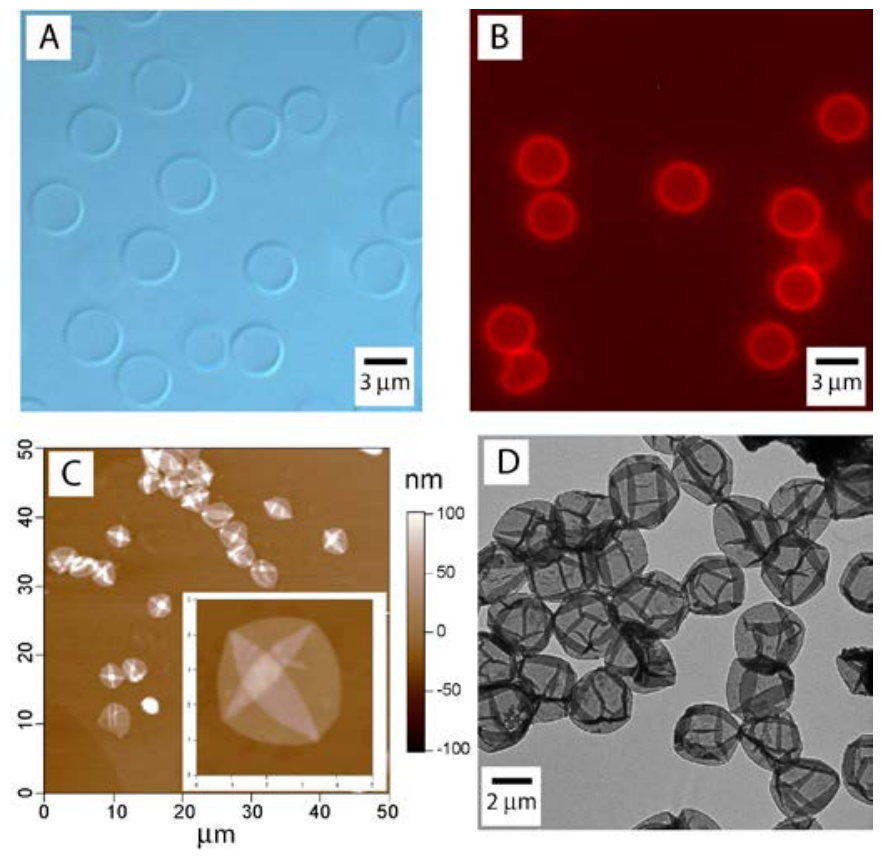

Figure 1. Microscopy images of $\left(\mathrm{PVPON}_{\mathrm{Alk}}\right)_{2} /\left(\mathrm{PGA}_{\mathrm{Alk}+\mathrm{DOx}}\right)_{3} /\left(\mathrm{PVPON}_{\mathrm{Alk}}\right)_{2}$ capsules obtained from $3 \mu \mathrm{m}$ diameter silica particles. (A) Differential interference contrast image; (B) fluorescence image; (C) AFM images for scanned areas of $50 \mathrm{x}$ $50 \mu \mathrm{m}^{2}$ and $5 \times 5 \mathrm{~m}^{2}$ (inset); (D) TEM image.

Previously, cell-based studies of MDR have involved establishing MDR cancer cell lines through drug selection ${ }^{[3,17]}$ or transfection with $\mathrm{ABC}$ transporter genes. ${ }^{[3,18]}$ To mimic acquired drug resistance occurring in cancer patients, LIM1899 cells were exposed to a low dose of DOX (50 nM) in a single-step selection, resulting in resistant cells (LIM1899MDR) that are capable of growth in the continuous presence of $50 \mathrm{nM}$ DOX. The dose of DOX used was based on reports that the steady-state plasma concentrations of DOX in patients after standard bolus infusions are $25-250 \mathrm{nM}^{\left[{ }^{[19]}\right.}$ Selection with a higher concentration of DOX (100 nM) was also performed, but the cells failed to survive under this regime, suggesting that LIM1899 cells are sensitive to DOX. Following selection, resistance to DOX in LIM1899MDR cells was evaluated using the MTT assay (Figure S1a). The $\mathrm{IC}_{50}$ value of DOX for LIM1899MDR cells was approximately $880 \mathrm{nM}$, which is 14-fold higher than the parental cells (Table 1). Interestingly, LIM1899MDR cells simultaneously developed a 16-fold crossresistance to PTX (Figure S1b, Table 1). DOX and PTX are both substrates of Pgp, ${ }^{[3]}$ but execute their cytotoxicity through very different mechanisms. ${ }^{[19,20]}$ This strongly suggests that overexpression of Pgp in LIM1899MDR cells may play a role in conferring the resistance. We therefore compared the cell surface expression of Pgp in LIM1899 and LIM1899MDR cells using flow cytometry (FC) and confocal laser scanning microscopy (CLSM) with an anti-Pgp monoclonal antibody. The parental cells display very little expression of Pgp on the cell membrane, consistent with the sensitivity to DOX, whereas the drug-resistant cells demonstrate significantly increased expression of Pgp (Figure S2).

To further understand the role of Pgp in MDR, we next characterized the cellular uptake and distribution of free DOX in both parental and resistant LIM1899 colorectal cancer cells. After exposure to $1 \mu \mathrm{M}$ DOX for $24 \mathrm{~h}$, the DOX-related fluorescence intensity was quantified using FC, and the intracellular localization was determined using CLSM. In the absence of Pgp, LIM1899 cells showed approximately a 2.5-fold higher total DOX accumulation than LIM1899MDR cells (Figure 2A). In addition, the expression of Pgp changed the pattern of DOX distribution, as most DOX was localized in the nuclei of the parental cells (Figure 2B), whereas significant cytoplasmic DOX accumulation was shown in the resistant cells (Figure 2C). Studies have shown that the ABC transporters are generally expressed on the plasma membrane to reduce the cellular accumulation of drugs. ${ }^{[21]}$ Some reports have also suggested that subcellular localization of the transporters can further actively sequester drugs away from the targets. ${ }^{[22]}$

One potential mechanism by which nanocarriers could enhance cellular accumulation of Pgp substrates is through endocytosis. As an initial test to verify the use of microcapsules for evasion of MDR, DOX-loaded PGA microcapsules (PGA-DOX capsules) were applied to the parental and resistant cells. Following treatment, the cellular accumulation of DOX was evaluated by FC. Figure 3A shows that DOX-related fluorescence intensity in LIM1899MDR cells was about 1.8-fold higher than LIM1899 cells, suggesting that the uptake and retention of DOX delivered by PGA capsules is enhanced in the resistant cells. It has been shown that the internalization of polyelectrolyte capsules (500 $\mathrm{nm}$ to $3 \mu \mathrm{m}$ in diameter) involves macropinocytosis, ${ }^{[11 \mathrm{~d}, 23]}$ which bypasses Pgp on the cell surface. Interestingly, recent studies have suggested that nanocarriers can activate endocytosis in drug resistant cells through their unique nanoscale properties. ${ }^{[10,24]}$ Potentially, the microcapsules may also stimulate endocytosis in LIM1899MDR cells. In contrast, the uptake of dextran (10 kDa), a marker for fluid phase endocytosis, was not significantly different between LIM1899 and LIM1899MDR cells (Figure S3). Further studies will be required to elucidate the interaction between PGA capsules and both cell lines, and reveal cellular changes after exposure to microcapsules. 
A
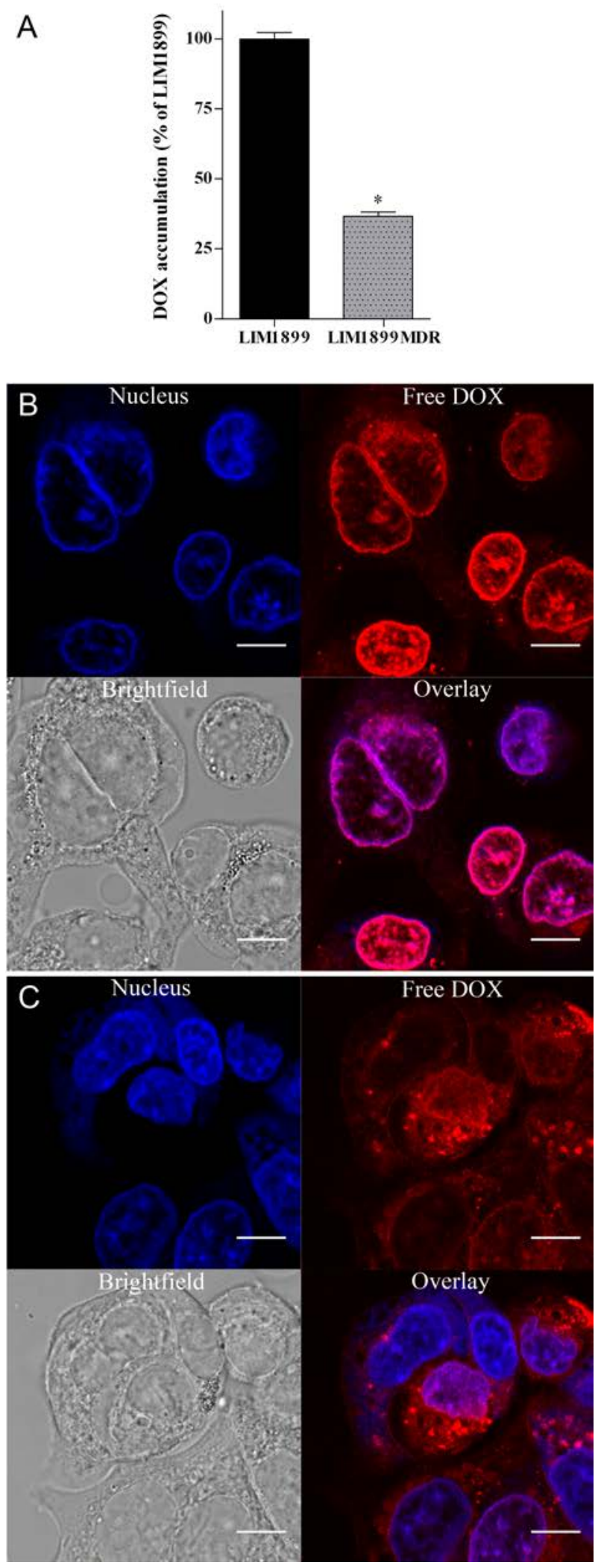

Figure 2. Comparison of intracellular accumulation and distribution of free DOX in LIM1899 and LIM1899MDR cells. Cells were treated with $1 \mu \mathrm{M}$ DOX for $24 \mathrm{~h}$. (A) Cellular accumulation of DOX quantified by flow cytometry and expressed as a percentage of LIM1899 cells. $\left.{ }^{*}\right)$ $p<0.0001$ (t-test). Intracellular distribution of DOX in LIM1899 (B) and LIM1899MDR (C) visualized by CLSM. Nuclei were counterstained with Hoechst 33342 (blue). Scale bars, $10 \mu \mathrm{m}$.
A
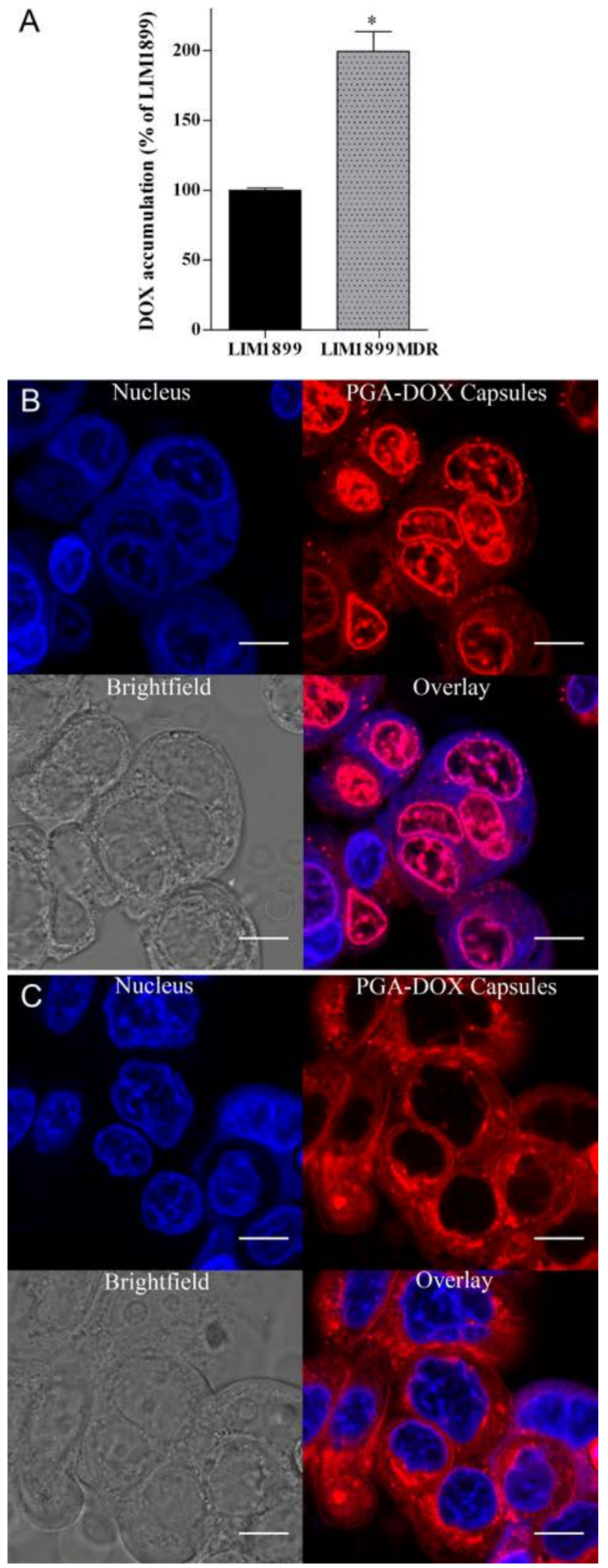

Figure 3. Comparison of intracellular accumulation and distribution of PGA-DOX capsules in LIM1899 and LIM1899MDR cells. Cells were treated with $3 \mu \mathrm{m}$ PGA-DOX capsules at a ratio (capsules to cells) of 100:1 for $24 \mathrm{~h}$. (A) Cellular accumulation of DOX quantified by flow cytometry and expressed as a percentage of LIM1899 cells. $\left({ }^{*}\right)$ $\mathrm{p}=0.0001$ (t-test). Intracellular distribution of DOX in LIM1899 (B) and LIM1899MDR (C) visualized by CLSM. Nuclei were counterstained with Hoechst 33342 (blue). Scale bars, $10 \mu \mathrm{m}$. 
Next, we examined the intracellular distribution of DOX delivered by PGA-DOX capsules using CLSM. In the parental cells, DOX-related fluorescence was predominantly localized in the nuclei, but also in the cytoplasm (Figure 3B), which is consistent with our previous report. ${ }^{[11 b]}$ In contrast, DOX-associated fluorescence was largely distributed in the cytoplasm in LIM1899MDR cells (Figure 3C). The substantially decreased nuclear DOX accumulation in LIM1899MDR cells is consistent with the characteristic distribution of DOX when Pgp-overexpressed cells are treated with either DOX in solution ${ }^{[25]}$ or DOX-loaded nanocarriers. ${ }^{[26]}$

Following the analysis of cellular uptake of PGA-DOX capsules, we evaluated the cytotoxicity of drug-loaded PGA capsules in both parental and resistant cancer cells using the MTT cell viability assay. Due to the modularity of the click-LbL approach, PGA capsules were loaded with either DOX or PTX by incorporation of PGADOX or PGA-PTX polymer-drug conjugates, respectively. By UVvis absorbance, we determined the drug loading capacity for both capsules (equivalent to $0.64 \mathrm{pg}$ of DOX per PGA-DOX capsule, and 0.48 pg of PTX per PGA-PTX capsule). To demonstrate the general applicability of the system, PGA-DOX or PGA-PTX capsules were used individually to challenge the MDR. The drug-loaded capsules were incubated with cells at various capsule-to-cell ratios (from 12.5 to 200:1) for 48 h. As shown in Figure S4, cell viability in both LIM1899 and LIM1899MDR cells was reduced in a doseresponsive manner when treated with either of the drug-loaded PGA capsules. Based on the amount of drug in each capsule and the dose of capsules used for each treatment, we calculated the apparent $\mathrm{IC}_{50}$ (Table 1). In our previous studies, we have shown significantly enhanced efficacy of DOX delivered by LbL-assembled submicron capsules compared to free drug. ${ }^{[11 a, 11 d]}$ The greater apparent IC $_{50}$ values of these drug-loaded PGA capsules compared to free drugs shown here are presumably due to the less efficient cellular uptake as a result of their large size ( $3 \mu \mathrm{m}$ in diameter) and low fouling PVPON corona layers. ${ }^{[16]}$ In attempts to increase the efficacy of PGA capsules, future studies will be aimed at functionalization of submicron-sized PGA capsule surfaces with targeting ligands to promote the specific cellular uptake. Moreover, we evaluated the bypass of Pgp in the resistant cells by comparing the resistance index (RI), which is determined by comparing $\mathrm{IC}_{50}$ values in LIM1899MDR versus LIM1899, when different agents were applied (Table 1). The RI of PGA-DOX capsules in LIM1899MDR cells (2.69) was significantly reduced compared to free DOX (14.15). Similarly, a reduction of RI was shown between PGA-PTX capsules (1.66) and free PTX (16). These data suggest that treatment with drug-loaded PGA capsules sensitizes the resistant cells.

Table 1. $I C_{50}$ values of free drugs and apparent $I C_{50}$ values of drugloaded PGA capsules, and corresponding resistance index in LIM1899 and LIM1899MDR cells.

\begin{tabular}{llll}
\hline Agents & $\begin{array}{l}\mathrm{IC}_{50}(\mathrm{nM}) \\
\mathrm{LIM1899}\end{array}$ & $\begin{array}{l}\mathrm{IC}_{50}(\mathrm{nM}) \\
\mathrm{LIM1899MDR}\end{array}$ & $\begin{array}{l}\text { Resistance } \\
\text { index }\end{array}$ \\
\hline Free DOX & $62.1 \pm 8.2$ & $878 \pm 250$ & 14.15 \\
$\begin{array}{l}\text { DOX-loaded } \\
\text { PGA capsule }\end{array}$ & $2013 \pm 1091$ & $5415 \pm 2361$ & 2.69 \\
$\begin{array}{l}\text { Free PTX } \\
\text { PrX-loaded }\end{array}$ & $237.5 \pm 1.4$ & $104.1 \pm 8.1$ & 16.00 \\
\begin{tabular}{l} 
PGA capsule \\
\hline
\end{tabular} & & $393.8 \pm 76.3$ & 1.66 \\
\hline
\end{tabular}


determined by acquisition of FL2. Cell viability was measured by reduction of 3-[4,5-dimethylthiazol-2-yl]-2,5-diphenyltetrazolium bromide (MTT), as described previously. ${ }^{[11 \mathrm{~d}]}$

Received: ((will be filled in by the editorial staff))

Published online on ((will be filled in by the editorial staff))

Keywords: polymer capsule $\cdot$ multidrug resistance $\cdot$ colorectal cancer - doxorubicin · paclitaxel

[1] D. B. Longley, P. G. Johnston, J. Pathol. 2005, 205, 275.

[2] S. G. Aller, J. Yu, A. Ward, Y. Wend, S. Chittaboina, R. Zhuo, P. M. Harrell, Y. T. Trinh, Q. Zhang, I. L. Urbatsch, G. Chang, Science 2009, 323, 1718.

[3] G. Szakács, J. K. Paterson, J. A. Ludwig, C. Booth-Genthe, M. M. Gottesman, Nat. Rev. Drug Discov. 2006, 5, 219.

[4] G. Szakács, J. Annereau, S. Lababidi, U. Shankavaram, A. Arciello, K. J. Bussey, W. Reinhold, Y. Guo, G. D. Kruh, M. Reimers, J. N. Weinstein, M. M. Gottesman, Cancer Cell 2004, 6, 129.

[5] a) S. B. Kaye, J. Clin. Oncol. 2008, 26, 2616; b) J. I. Fletcher, M. Haber, M. J. Henderson, M. D. Norris, Nat. Rev. Cancer 2010, 10, 147.

[6] J. R. Heath, M. E. Davis, Annu. Rev. Med. 2008, 59, 251.

[7] Y. Liu, L. Huang, F. Liu, Mol. Pharm. 2010, 7, 863.

[8] R. Li, R. Wu, L. Zhao, M. Wu, L. Yang, H. Zou, ACS Nano 2010, 4, 1399.

[9] a) X. Xiong, Z. Ma, R. Lai, A. Lavasanifar, Biomaterials 2010, 31, 757; b) M. Susa, A. K. Iyer, K. Ryu, F. J. Hornicek, H. Mankin, M. M. Amiji, Z. Duan, BMC Cancer 2009, 9, 399.

[10] X. Liang, H. Meng, Y. Wang, H. He, J. Meng, J. Lu, P. C. Wang, Y. Zhao, X. Gao, B. Sun, C. Chen, G. Xing, D. Shen, M. M. Gottesman, Y, Wu. J. Yin, L. Jia, Proc. Natl. Acad. Sci. U.S.A. 2010, 107, 7449.

[11] a) S. Sivakumar, V. Banal, C. Cortez, S-. F. Chong, A. N. Zelikin, F. Caruso, Adv. Mater. 2009, 21, 1820; b) C. J. Ochs, G. K. Such, Y. Yan, M. P. van Koeverden, F. Caruso, ACS Nano 2010, 4, 1653; c) L. Hosta-Rigau, B. Städler, Y. Yan, E. C. Nice, J. K. Heath, F. Albericio, F. Caruso, Adv. Funct. Mater. 2010, 20, 59; d) Y. Yan, A. P. R. Johnston, S. J. Dodds, M. M. J. Kamphuis, C. Ferguson, R. G. Parton, E. C. Nice, J. K. Heath, F. Caruso, ACS Nano 2010, 4, 2928; e) Y. Wang, Y. Yan, J. Cui, L. Hosta-Rigau, J. K. Heath, E. C. Nice, F. Caruso, Adv. Mater. DOI 10.1002/adma.201001497.
[12] S. M. Andrew, J. G. Teh, R. W. Johnstone, S. M. Russell, R. H. Whitehead, I. F. McKenzie, G. A. Pietersz, Cancer Res. 1990, 50, 5225.

[13] G. K. Such, E. Tjipto, A. Postma, A. P. R. Johnston, F. Caruso, Nano Lett. 2007, 7, 1706

[14] P. Rivera-Gil, S. De Koker, B. G. De Geest, W. J. Parak, Nano Lett. 2009, 9, 4398.

[15] C. Cortez, E. Tomaskovic-Crook, A. P. R. Johnston, B. Radt, S. H. Cody, A. M. Scott, E. C. Nice, J. K. Heath, F. Caruso, Adv. Mater. 2006, 18, 1998.

[16] C. R. Kinnane, G. K. Such, G. Antequera-García, Y. Yan, S. J. Dodds, L. M. Liz-Marzan, F. Caruso, Biomarcomolecules 2009, 10, 2839.

[17] A. M. Calcagno, J. M. Fostel, K. K. W. To, C. D. Salcido, S. E. Martin, K. J. Chewning, C. Wu, L. Varticovski, S. Bates, N. J. Caplen, S. V. Ambudkar, Br. J. Cancer 2008, 98, 1515.

[18] F. Shen, S. Chu, A. K. Bence, B. Bailey, X. Xue, P. A. Erickson, M. H. Monstrose, W. T. Beck, L. C. Erickson, J. Pharmacol. Exp. Ther. 2008, 324, 95.

[19] G. Minotti, P. Menna, E. Salvatorelli, G. Cairo, L. Gianni, Pharmacol. Rev. 2004, 56, 185.

[20] M. A. Jordan, L. Wilson, Nat. Rev. Cancer 2004, 4, 253.

[21] B. D. Stride, S. P. Cole, R. G. Deeley, J. Biol. Chem. 1999, 274, 22877.

[22] S. Meschini, A. Calcabrini, E. Monti, D. Del Bufalo, A. Stringaro, E. Dolfini, G. Arancia, Int. J. Cancer 2000, 87, 615.

[23] a) B. G. De Geest, R. E. Vandenbroucke, A. M. Guenther, G. B. Sukhorukov, W. E. Hennink, N. N. Sanders, J. Demeester, S. C. De Smedt, Adv. Mater. 2006, 18, 1005; b) A. M. Javier, O. Kreft, M. Semmling, S. Kempter, A. G. Skirtach, O. T. Bruns, P. Del Pino, M. F. Bedard, J. Rädler, J. Kas, C. Plank, G. B. Sukhorukov, W. J. Parak, Adv. Mater. 2008, 20, 4281; c) S. De Koker, B. G. De Geest, S. K. Singh, R. De Rycke, T. Naessens, Y. Van Kooyk, J. Demeester, S. C. De Smedt, J. Grooten, Angew. Chem. Int. Ed. 2009, 48, 8485.

[24] a) X. Dong, C. A. Mattingly, M. T. Tseng, M. J. Cho, Y. Liu, V. R. Adams, R. J. Mumper, Cancer Res. 2009, 69, 3918; b) H. C. Kang, O. Samsonova, Y. H. Bae, Biomaterials 2010, 31, 3071.

[25] A. Rajagopal, S. M. Simon, Mol. Biol. Cell 2003, 14, 3389.

[26] M. D. Chavanpatil, A. Khdair, B. Gerard, C. Bachmeier, D. W. Miller, M. P. V. Shekhar, J. Panyam, Mol. Pharm. 2007, 4, 730.

[27] C. Li, D. Yu, R. A. Newman, F. Cabral, L. C. Stephens, N. Hunter, L. Milas, S. Wallace, Cancer Res. 1998, 58, 2402. 
Entry for the Table of Contents (Please choose one layout)

Layout 1:

\section{Evasion of Multidrug}

Resistance

Yan Yan, Christopher J. Ochs, Georgina K. Such, Joan K. Heath, Edouard C. Nice, and Frank Caruso*

Drug-Loaded Biodegradable Polymer Capsules to Bypass P-GlycoproteinMediated Multidrug Resistance in Colorectal Cancer Cells
Biodegradable polymer capsules with drug-loaded multilayers were prepared

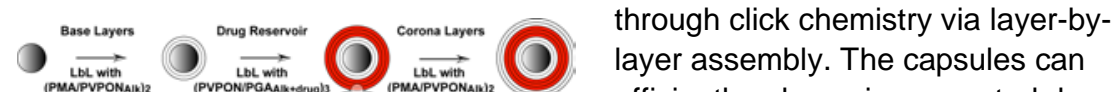
efficiently release incorporated drug by intracellular enzymatic degradation in both sensitive and resistant colorectal cancer cells in vitro. The endocytosis of drug-loaded capsules leads to bypass of the Pgp-mediated multidrug resistance in cancer cells. 


\section{University Library}

\section{- M M N E R VA A gateway to Melbourne's research publications}

Minerva Access is the Institutional Repository of The University of Melbourne

Author/s:

Yan, Y;Ochs, CJ;Such, GK;Heath, JK;Nice, EC;Caruso, F

Title:

Bypassing Multidrug Resistance in Cancer Cells with Biodegradable Polymer Capsules

Date:

2010-12-14

Citation:

Yan, Y., Ochs, C. J., Such, G. K., Heath, J. K., Nice, E. C. \& Caruso, F. (2010). Bypassing Multidrug Resistance in Cancer Cells with Biodegradable Polymer Capsules. ADVANCED MATERIALS, 22 (47), pp.5398-+. https://doi.org/10.1002/adma.201003162.

Persistent Link:

http://hdl.handle.net/11343/123323 